\title{
THE MAGNETIC FIELD IN M33
}

U.R. BUCZILOWSKI, R. BECK

Max-Planck-Institut für Radioastronomie

Auf dem Hügel 69

D-5300 Bonn 1, F.R.G.

Measurements at five radio wavelengths between 2.8 and $21.1 \mathrm{~cm}$ (Buczilowski and Beck, 1987) with the Effelsberg 100-m telescope make M33 the best studied nearby galaxy in linear polarization so far. The polarized emission is concentrated to the northwestern quairant of the galaxy. Almost no polarization is detectable around the 35 known supernova remnant candidates. The explosions may have disturbed the interstellar magnetic field on scale sizes smaller than our antenna beams.

Compared with other spiral galaxies the magnetic fjeld properties for M33 are remarkable: using either the minimum energy requirement or the internal rotation measures we find a total magnetic field strength of $\mathrm{B}_{\mathrm{t}}=4.5^{ \pm} 1.4 \mu \mathrm{G}$. This represents one of the weakest fields in the sample of 8 spiral galaxies studied so far; values in the range $9 \pm 4 \mu \mathrm{G}$ are usually found.

From the degree of linear polarization $(11 \pm 5 \%)$ of synchrotron emission at $6.3 \mathrm{~cm}$ we obtain a ratio between the strength of the uniform and random magnetic field of $B_{u} / B_{r}=0.4 \pm 0.1$ over the scale of the resolution element of $510 \times 880 \mathrm{pc}$. M33 has the lowest field uniformity of 7 spiral galaxies for which comparable estimates are available.

We have averaged the distribution of unambiguous rotation measures obtained from the $6.3,11.1$ and $17.4 \mathrm{~cm}$ data azimuthally in two rings in the plane of M33. Double-periodical fits reveal a low internal rotation measure of $15 \pm 5 \mathrm{rad} / \mathrm{m}^{2}$, less than half the value for other galaxies. Following Tosa and Fujimoto (1978) we calculated from the fit parameters a bisymmetric model projected onto the sky. The orientations of the magnetic field can be determined from the $6.3 \mathrm{~cm}$ data after correction for the foreground Faraday rotation. A comparison of model and observations shows coincidences of the expected maximal field strength with the area of high polarization in the north as well as of the neutral lines with the areas of low polarization to the east and west. We conclude that the polarization data is compatible with a bisymmetric magnetic field although more sophisticated models would be desirable.

\section{References}

Buczilowski, U.R. and Beck, R. (1987), Astron. Astrophys. Suppl. 68, $171-185$.

Tosa, M. and Fujimoto, M. (1978), Publ. Astron. Soc. Japan 30, 315-325. 210

R. Beck et al. (eds.), Galactic and Intergalactic Magnetic Fields, 210.

(c) 1990 IAU. Printed in the Netherlands. 